\title{
El plan de estudios y el perfil profesional del egresado de la Maestría en Agronegocios de la Escuela de Postgrado de la Universidad Nacional Agraria La Molina
}

\author{
The curriculum and the professional profile of its graduates of the Master of Agribusiness of the \\ Postgraduate School of the Universidad Nacional Agraria La Molina
}

\author{
Roberto José Morales Muñoz
}

\begin{abstract}
Resumen
En la educación superior, no es evaluado el perfil profesional de los egresados de las Maestrías, así como los requerimientos para su futuro desempeño profesional. Este vacío es aprovechado por el presente trabajo cuyo propósito fue determinar la relación existente entre el plan de estudios de la Maestría en Agronegocios de la Universidad Nacional Agraria La Molina (UNALM) y el perfil profesional de sus egresados, en virtud al nivel de congruencia, continuidad e integración del plan de estudios, en relación al perfil del egresado. La investigación es cuantitativa, la metodología es descriptivacorrelacional. Se utilizaron las pruebas de contraste de Shapiro-Wilk, Chi-cuadrado y Kolmogorov-Smirnov, así como el coeficiente de correlación de Spearman y el criterio de confiabilidad Alfa de Cronbach para validar los datos recogidos en el cuestionario repetitivo, cerrado y estructurado, en una población de 77 egresados cuya muestra fue de 15 . Los resultados confirman la existencia de una relación altamente significativa entre el plan de estudios y el perfil profesional del egresado y un nivel altamente significativo de congruencia, continuidad e integración entre el plan de estudios de la Maestría, en función al perfil profesional del egresado.
\end{abstract}

Palabras clave: plan de estudios; perfil profesional del egresado; diseño curricular; nivel de congruencia; nivel de continuidad; nivel de integración.

\begin{abstract}
Asbtract
In higher education, the professional profile of the Master's degree graduates is not evaluated, as well as the requirements for their future professional performance. The present research, takes advantage of this gap, whose purpose was to determine the relationship between the curriculum of the Master of Agribusiness of Universidad Nacional Agraria La Molina (UNALM) and the professional profile of its graduates, according to the level of congruence, continuity and integration of the curriculum, in relation to graduate's profile. The research is quantitative; the methodology is descriptive-correlational. We used the Shapiro-Wilk test, Chi-square and Kolmogorov-Smirnov tests as well as the Spearman correlation coefficient and Cronbach's Alpha reliability criterion to validate the data collected in the repetitive, closed and structured questionnaire in a population of 77 graduates whose sample was 15 graduates. The results confirm the existence of a highly significant relationship between the curriculum and the professional profile of its graduates and a highly significant level of congruence, continuity and integration between the curriculum of the Master's degree, according to the professional profile of the graduate.
\end{abstract}

Key words: curriculum; graduate's professional profile; curricular design; level of congruence; level of continuity; level of integration.

\section{Introducción}

La educación actual requiere fundamentos sólidos que permitan optimizar sus procesos educativos desde la perspectiva científica, social, y humanística, sin olvidar el campo ocupacional. Albornoz (1991), plantea que la educación debe considerarse como un proceso continuo y sistemático en la formación integral de un individuo apto para responder a las exigencias espacio - temporal que la presente época amerita. En la educación superior peruana existe la necesidad de realizar evaluaciones periódicas de su funcionamiento. Castro (1984), plantea la necesidad de realizar una evaluación integral de las instituciones de educación superior en el país, lo que obliga a proponer modelos de evaluación integral, que incluyen comprobar la factibilidad de los programas educativos de post grado. El esquema de planificación para la evaluación curricular en instituciones de educación superior está vinculado con la conceptualización y contemporaneidad del currículo que se utiliza. Ello permitirá establecer la relación teoría -praxis más efectiva. Los planes de estudio de las universidades refieren a los proyectos formativos que ofrecen para acreditar a los profesionales egresados de ellas. En tal sentido, los currículos que se elaboran como proyecto de formación integral deben responder a las necesidades 
de la empresa y la sociedad, a fin de contar con personal capacitado que afronte con mayor eficacia y eficiencia los retos futuros. El perfil del egresado debe responder adecuadamente a los avances tecnológicos y al proceso de globalización, multiplicando valores, actitudes y conocimientos que afecten positivamente al entorno donde se desempeñan los egresados. La UNALM ofrece diversas maestrías, entre ellas, la Maestría en Agronegocios la cual brinda a cualquier profesional capaz y ajeno al tema de gestión de agronegocios la posibilidad de capacitarse en una especialización integral, novedosa y trascendental. El plan de estudios de la Maestría debe estar en armonía y cumplir la misión y visión institucional y las expectativas del egresado, atender su formación y futuro desempeño de sus funciones. El presente trabajo tuvo como objeto establecer la relación el plan de estudios de la Maestría en Agronegocios de la UNALM y el perfil profesional de sus egresados, al determinar el nivel de congruencia, continuidad e integración del plan de estudios de la Maestría de la Universidad rectora del tema agrario. En la búsqueda de antecedentes, se encontraron investigaciones de obligatoria revisión, a fin de entender y consolidar el objeto de estudio.

Pereira (2012), analizó el nivel de formación de los graduados para determinar un perfil profesional de los estudiantes y su contribución a la formación de los titulados técnicos agrícolas. Llanos (2010), investigó sobre el nivel de relación entre el perfil profesional y el plan de estudios de la Facultad de Ciencias de la Educación de la Universidad Nacional Hermilio Valdizán de Huánuco, encontrando la existencia de una relación significativa, entre las variables plan de estudios con el desempeño docente y el perfil profesional con desempeño docente, en los egresados de la especialidad. Barrientos y Vildoso (2007), analizaron las variables perfil académico, plan de estudios y nivel de conocimiento de investigación en los alumnos de maestrías de la Escuela de Postgrado de la Facultad de Educación, encontrando una correlación significativa entre el plan de estudios y el nivel de conocimiento de investigación, entre el perfil académico y el nivel de conocimiento de investigación y, finalmente, entre el perfil académico y el plan de estudios en el nivel de conocimiento de investigación. Ramírez y Tovar (2005), analizaron la situación de los Enfermeros egresados de las especializaciones en Enfermería MaternoPerinatal, Nefrológica y Salud Familiar, en relación a las características de sus empleos, condiciones actuales y logros como especialistas. Rodríguez del Solar (1997) comprobó las causas de algunas deficiencias significativas del currículo y del perfil profesional del Licenciado de la Facultad de Educación de la UNMSM.

\section{La teoría curricular}

La educación en términos de resultados, que se pretende alcanzar en función a la formación del ciudadano y del tipo de sociedad que se desea construir, se concreta mediante el currículo, qué actúa como proceso operativo en el que ingresan un conjunto de elementos como: actores sociales, objetivos, recursos, etc., que interactúan entre sí para alcanzar las metas educativas trazadas. En ese sentido, mediante la planificación y ejecución del currículo se fortalece el logro del tipo de hombre y sociedad que un estado desea para su sistema educativo.

Las teorías sobre el currículo evolucionan, son dinámicas y suelen significar un marco de discusión para fundamentar y justificar lo que se enseña y cómo se hace. El concepto de currículo es dinámico e incorpora una serie de elementos que no se visualizan en forma aislada sino en sus mutuas relaciones. Franklin Bobbitt en sus obras "The Curriculum" y "How to make a Curriculum", inicia tendencias acogidas por muchos autores, tales como: Tyler, Taba, Rule, Díaz, Arredondo, Lafrancesco, entre otros. Bobbit considera que el criterio para determinar el contenido que debe formar parte de un plan de estudios se realizará a partir de las exigencias que en la vida adulta se le harán al individuo para que tenga un buen desempeño social, considerando las demandas de los empleadores. Según Salinas (1994), la influencia más importante del trabajo de Bobbitt consiste en la serie de supuestos que planteó y se mantienen vigentes en la actualidad, manteniendo sus propuestas en la enseñanza.

Para Álvarez de Zayas (2001), “el currículo constituye el plan de acción para la formación del profesional, a partir del cual se organiza, dirige, ejecuta y controla el proceso de enseñanza aprendizaje, para la formación de los recursos humanos que requiere la sociedad, teniendo en cuenta las necesidades del contexto social y los intereses y motivaciones de los actores principales del proceso". En otras palabras, el currículo forma recursos humanos en cualquier campo. El plan de estudios es el medio que permite seleccionar y organizar los conocimientos con criterios de secuencialidad y continuidad considerados como fundamentales para el logro del perfil académico profesional.

\section{El diseño curricular}

No existe un solo enfoque del diseño curricular capaz de dar respuesta integral a la diversidad de problemas que plantea el currículo y su praxis. En la literatura revisada sobre el diseño curricular es recurrente encontrar confusiones y ambigüedad en los modelos curriculares. Kissack (1998) también considera que se mezclan currículo con el plan de estudios y programas.

Álvarez de Zayas (2001) plantea la necesidad de profundizar, reflexionar e indagar acerca de cómo diseñar, ejecutar y evaluar un currículo con la concepción que desarrolle una nueva mentalidad para la actuación práctica del profesional, donde armonicen todos los integrantes del sistema en concordancia con los cambios del paradigma actual, y teniendo en cuenta el entorno social, constituyen una de las funciones de las instituciones universitarias en el mundo, en nuestro país, y de todos los responsables en el funcionamiento de los procesos que en ella se llevan a cabo. Además de esto, el diseño debe ser evaluado y 
actualizado permanentemente, como lo sostiene Tyler (1973), "la evaluación curricular debe ser entendida como una actividad sistemática y permanente que permita mejorar en forma continua el currículo, ya que el mismo puede dejar de responder a las necesidades y valores que lo justifican".

Zabalza (2012) plantea que el proyecto curricular es como una partitura que, como documento, concreta y fija la propuesta que se plantea y se evita así que cada músico ceda a la tentación de crear su propia melodía y convertir el proceso en un caos. Ante esta situación, el currículo debe estar en permanente construcción y en permanente evaluación, atendiendo a las necesidades de la sociedad.

\section{El plan de estudios}

El plan de estudios es el diseño curricular concreto respecto a determinadas enseñanzas realizadas por una universidad, sujeto a directrices generales comunes y las propias universitarias, cuya superación da derecho a la obtención de un título de grado oficial y validez en todo el territorio nacional. Álvarez de Zayas (2001) agrega que dentro de los documentos del diseño curricular está el plan de formación profesional, plan de estudio o pensum, documento en cual se diseñan las características más importantes de proceso docente-educativo a nivel de carrera.

El plan de estudios es un instrumento dinámico, flexible y refleja las metas y experiencias educativas a ser alcanzadas y proporcionadas, respectivamente, para lograr ese fin. En el ámbito universitario, cada institución elabora sus propios planes de estudio, los mismos que deben estar en permanente actualización; por ello, se requiere una metodología de evaluación curricular que incluya diversos elementos de evaluación. En Díaz (1993), se encuentra una alternativa que integra los elementos de evaluación interna y externa.

\section{El perfil profesional del egresado}

La tarea universitaria es formar al profesional al nivel de egresado, de maestría o doctorado bajo el concepto de profesional básico, magister o doctor, según sea el caso.

El perfil profesional es el conjunto de rasgos y capacidades que, certificadas apropiadamente por la Universidad, permite que alguien sea reconocido por la sociedad como profesional, encomendándole tareas para las que está capacitado y es competente.

Díaz (1981) plantea que el perfil profesional lo componen tanto conocimientos y habilidades como actitudes. Estos conceptos, similares entre sí, delimitan operativamente el ejercicio profesional. Se concibe al perfil profesional como la determinación de acciones propias generales y específicas que se espera sean logrados por los estudiantes al término del proceso educativo en las áreas o campos de acción, tendientes a la solución de las necesidades sociales. Por tanto, una evaluación del perfil debe generarse a partir de los elementos que lo definen, de su congruencia y continuidad con la etapa de la fundamentación del proyecto curricular, así como su vigencia.

El perfil de formación describe los rasgos que definirán al egresado de una carrera. El perfil profesional debe identificar un conjunto de conocimientos, capacidades, habilidades, aptitudes y actitudes que debe poseer el egresado para un excelente desempeño profesional. Asimismo, la metodología para elaborar un perfil profesional está vinculada a las características generales de la profesión y su práctica.

\section{Materiales y métodos}

La hipótesis general plantea la existencia de una relación entre el plan de estudios y el perfil profesional del egresado de la Maestría en Agronegocios de la UNALM entonces impactará favorablemente en el logro de los conocimientos, actitudes y valores y habilidades del egresado de la Maestría. Las hipótesis específicas plantean la existencia de un nivel alto de congruencia, de continuidad y de integración del plan de estudios de la Maestría en Agronegocios de la UNALM, en función del perfil de su egresado.

Para desarrollar la prueba de hipótesis se operacionalizó el nivel de abstracción de las variables y el manejo de sus referentes empíricos. Cada variable se descompuso en indicadores, sobre estudios anteriores, considerándose la variable dependiente (perfil profesional del egresado) y la variable independiente (plan de estudios). Se siguió la metodología de evaluación curricular construida por Díaz (1993), integrando elementos de evaluación interna (pan de estudios) y externa (perfil profesional del egresado).

A efectos del presente estudio, se planteó un análisis institucional, como responsable del funcionamiento de los procesos que en ella se llevan a cabo, generando las dimensiones en las que se debe encontrar inmerso el perfil profesional del egresado como: objetivos institucionales, seguimiento a los graduados y el perfil publicado.

Finalmente, el instrumento utilizado fue el cuestionario repetitivo, cerrado y estructurado, el cual midió la variable el plan de estudios analizó los indicadores descritos en la Tabla 1, según las dimensiones aspecto formativo, aspecto actitudinal y personal, y el aspecto cognitivo. Asimismo, el instrumento utilizado fue el cuestionario repetitivo, cerrado y estructurado, el cual midió la variable perfil del egresado analizó los indicadores descritos en la Tabla 1, según las dimensiones objetivos institucionales, seguimiento a graduados y perfil publicado.

\section{Tipo de investigación}

La investigación es cuantitativa, descriptiva-correlacional. Se recolectaron, midieron y evaluaron los datos sobre diversos conceptos (variables), aspectos, dimensiones o componentes del fenómeno investigado, siguiendo a Hernández, R (1996) y Hernández, Fernández y Baptista (2006). 


\section{Método de la investigación}

El trabajo se basó en el método hipotético-deductivo, con predomino del enfoque cuantitativo y uso de estadística no paramétrica. Los datos obtenidos se cuantificaron mediante técnicas cuantitativas. La población estuvo conformada por 10 promociones de egresados de la Maestría (77 egresados) que han cumplido con todos los requisitos. La muestra es no probabilística intencionada compuesta por 15 egresados del año 2013, bajo criterios de inclusión y exclusión. El estudio utilizó el muestreo por juicio o a criterio del investigador, siguiendo a Arista (1984) por ser una muestra expeditiva y no interviene el azar. Se utilizó la adecuación de Shapiro Wilk para determinar la muestra. El instrumento utilizado fue el cuestionario repetitivo, cerrado y estructurado como parte de la técnica de la encuesta, con preguntas en positivo y negativo, aplicadas mediante el envío por correo electrónico a cada egresado. Se utilizó "la validez de criterio porque las preguntas están referidas a un patrón de medida o criterio externo", según Hernández (1996). Además, se utilizó el análisis documental, la observación directa, continuada y sistemática.

El error muestral es de $+/-5 \%$ y el nivel de confianza: 95\%. El procedimiento adoptado para la muestra fue el criterio selectivo por la uniformidad en los sílabos, docentes con experiencia nacional e internacional y de campo, así como la evaluación por competencias. Se procesó la información mediante el SPSS, versión 17.0. Se asignaron valores a las respuestas del cuestionario, según la escala de Likert.

La prueba de inferencia utilizada fue la prueba Chicuadrado $\left(X^{2}\right)$, siguiendo a Pagano (1999). La distribución $\mathrm{X}^{2}$ se obtuvo con grados de libertad G.L $=\left(\mathrm{N}^{\circ}\right.$ de filas - 1$)$ $\left(\mathrm{N}^{\mathrm{o}}\right.$ de columnas -1$)$. No habrá valores negativos. El valor mínimo fue 0 . Todas las curvas son asimétricas. Cuando aumenten los grados de libertad las curvas serán menos elevadas y más extendidas a la derecha. La prueba Chicuadrado se calculó a través de una Tabla de contingencia o tabulación cruzada, que consistió en una Tabla de dos dimensiones y cada dimensión contiene una variable.

\section{La contrastación de hipótesis}

Para saber si el valor de $X^{2}$ es o no significativo, se calcularon los grados de libertad. G.L. $=\left(\mathrm{N}^{\mathrm{o}}\right.$ de filas - 1) $\left(\mathrm{N}^{\mathrm{o}}\right.$ de columnas - 1). Luego, se acudió a la Tabla de distribución de Chi-cuadrado, eligiendo el nivel de confianza $(\mathrm{a}=0,05$ ó a $=0,01)$. Si el valor obtenido de $X^{2}$ fue igual o superior al valor de la "Tabla", se concluyó que las variables estuvieron relacionadas o no son independientes.

El nivel de confianza utilizado fue de $a=0,05$. La fórmula para obtener el $X^{2}$ obtenido fue la siguiente:

$$
\chi_{c}^{2}=\sum\left[\frac{\left(O_{i}-E_{i}\right)^{2}}{E_{i}}\right]
$$

$\mathrm{X}^{2}$ obtenido ${ }^{3} \mathrm{X}^{2}$ crítico entonces las variables no fueron independientes; es decir existió una relación. $\mathrm{X}^{2}$ obtenido ${ }^{3}$ $\mathrm{X}^{2}$ crítico entonces se rechazó la hipótesis nula $\left(\mathrm{H}_{0}\right)$, y por lo tanto se aceptó la hipótesis alterna $\left(\mathrm{H}_{1}\right)$.

El análisis de la significancia estadística de los coeficientes de Chi-cuadrado se sistematizó con la información del cuestionario mediante el sistema SPSS 17, cuyos resultados totales fueron determinados por variable según la encuesta aplicada. Luego, se especificaron las frecuencias observadas y se aplicó la fórmula para obtener las frecuencias esperadas. Se estableció una Tabla de frecuencias esperadas a partir de las frecuencias observadas.

Tabla 1. Operacionalización de variables

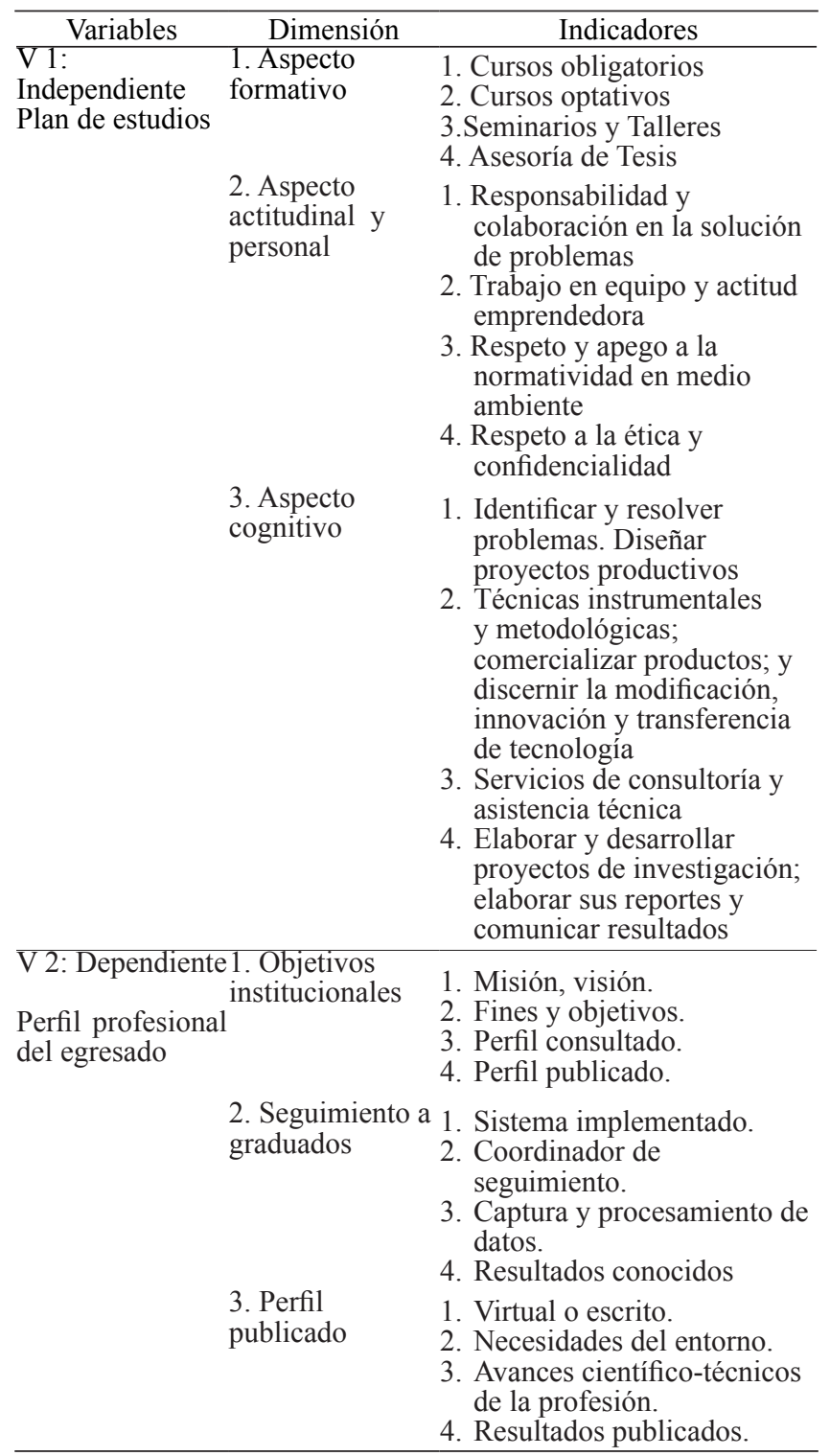

La frecuencia esperada de cada celda, casilla o recuadro, se calculó mediante la siguiente fórmula aplicada a la Tabla de frecuencias observadas:

$\mathrm{N}=$ Es el número total de frecuencias observadas.

$\mathrm{E}=$ (marginal del reglón) (marginal de columna) / N.

$$
\text { F. Esperada }=\frac{\text { Total de Fila } \times \text { Total de Columna }}{\text { Total General }}
$$

Finalmente se calculó el $X^{2}$ obtenido para poder contrastarlo con el $X^{2}$ crítico, de acuerdo a la Tabla del cálculo del Chi- 
cuadrado (Cuadro N.) y hacer la respectiva contrastación. El criterio de confiabilidad del instrumento se determinó por el coeficiente de Alfa Cronbach. Se utilizó el siguiente criterio de confiabilidad de valores: No es confiable ( 1 a 0 ), Baja confiabilidad (0.01 a 0.49), Moderada confiabilidad (0.5 a 0.75), Fuerte confiabilidad (0.76 a 0.89), Alta confiabilidad (0.9 a 1$)$.

Antes de realizar la prueba de hipótesis se usó la prueba de normalidad de Kolmogorov-Smirnov para establecer sí los instrumentos obedecerán a la estadística paramétrica o no paramétrica. Al ser la muestra inferior a 50 unidades se utilizó la adecuación de Shapiro Wilk para determinar la muestra.

Tabla 2. Prueba de normalidad de Shapiro-Wilk

\begin{tabular}{lccc}
\hline & Estadístico & Gl & Sig. \\
\hline $\begin{array}{lccc}\text { Variable: Perfil profesional del } \\
\text { egresado }\end{array}$ &, 801 & 15 &, 004 \\
Variable: Plan de estudios &, 766 & 15 &, 001 \\
\hline
\end{tabular}

Conclusiones de la prueba de normalidad. Las variables presentan distribución asimétrica. Por ello, para efectuar la prueba de hipótesis de alcance correlacional se so el estadígrafo de Spearman, el más exhaustivo para determinar la correlación entre variables.

\section{Resultados y discusión.}

Luego de someter las variables a la prueba de hipótesis, en función a la herramienta de levantamiento de información, se encontró lo siguiente:

Hipótesis planteada: existe relación entre el plan de estudios y el perfil profesional del egresado de la Maestría en Agronegocios de la UNALM que impacte favorablemente en el logro de los conocimientos, actitudes y valores, y habilidades en la comunidad educativa.

Hipótesis Nula: no existe relación entre el plan de estudios y el perfil profesional del egresado de la Maestría en Agronegocios de la UNALM que impacte favorablemente

Tabla 3. Variables: perfil profesional del egresado y plan de estudios

\begin{tabular}{|c|c|c|c|c|c|c|c|c|c|c|}
\hline \multirow{3}{*}{$\begin{array}{l}\text { Variable: } \\
\text { Perfil } \\
\text { profesional } \\
\text { del egresado }\end{array}$} & \multicolumn{8}{|c|}{ Variable: Plan de Estudios } & \multirow{2}{*}{\multicolumn{2}{|c|}{ Total }} \\
\hline & \multicolumn{2}{|c|}{$\begin{array}{c}\text { En } \\
\text { desacuerdo }\end{array}$} & \multicolumn{2}{|c|}{ Aceptable } & \multicolumn{2}{|c|}{ De acuerdo } & \multicolumn{2}{|c|}{$\begin{array}{l}\text { Totalmente de } \\
\text { acuerdo }\end{array}$} & & \\
\hline & $\mathrm{f}$ & $\%$ & $\mathrm{f}$ & $\%$ & $\mathrm{f}$ & $\%$ & $\mathrm{f}$ & $\%$ & f & $\%$ \\
\hline En desacuerdo & 1 & $6,7 \%$ & 0 &, $0 \%$ & 0 &, $0 \%$ & 0 &, $0 \%$ & 1 & $6,7 \%$ \\
\hline Aceptable & 0 &, $0 \%$ & 5 & $33,3 \%$ & 3 & $20,0 \%$ & 0 &, $0 \%$ & 8 & $53,3 \%$ \\
\hline De acuerdo & 0 &, $0 \%$ & 0 &, $0 \%$ & 5 & $33,3 \%$ & 1 & $6,7 \%$ & 6 & $40,0 \%$ \\
\hline Total & 1 & $6,7 \%$ & 5 & $33,3 \%$ & 8 & $53,3 \%$ & 1 & $6,7 \%$ & 15 & $100,0 \%$ \\
\hline $\begin{array}{l}\text { Chi-cuadrado d } \\
\text { Correlación de }\end{array}$ & 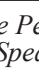 & $\begin{array}{l}\text { on }=2 \\
\text { an }=.7\end{array}$ & & g.l. $=$ & & .05 & & & & \\
\hline
\end{tabular}

en el logro de los conocimientos, actitudes y valores, y habilidades en la comunidad educativa. En consecuencia: de la UNALM.
La Tabla 3 demuestra, que el perfil profesional del egresado está relacionado directamente con el plan de estudios en los egresados de la Maestría de Agronegocios de la UNALM, según la correlación de Spearman de 0.749, representando una aceptable asociación de las variables y altamente significativa. Además, la prueba Chi-cuadrado $(21.797 * * * p<.05)$ es altamente significativa. Por tanto, se acepta la relación entre el plan de estudios y el perfil profesional del egresado de la Maestría en Agronegocios

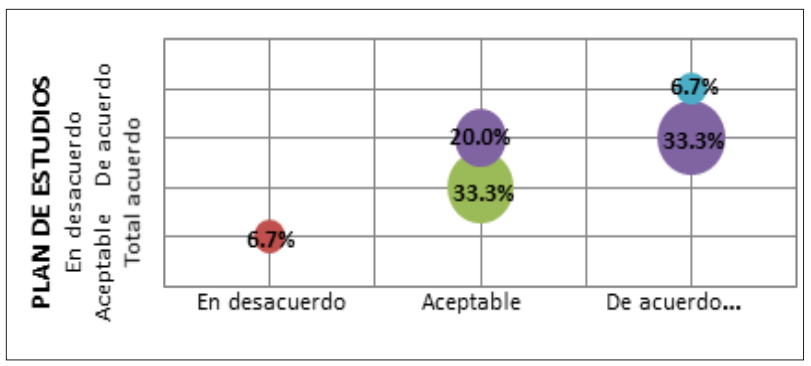

Figura 1. Variables: Perfil profesional del egresado y plan de estudios

Nota: en base a cuestionarios aplicados a la muestra del estudio, en el año 2013.

Se aprecia en la Figura 1, que el 6.7\% de los encuestados afirmó no estar de acuerdo con el perfil del profesional egresado y estar en desacuerdo con el plan de estudios de la Maestría de Agronegocios. Por otro lado, el 53.3\% afirmó, que es aceptable el perfil del profesional del egresado; de estos, el $33.3 \%$ planteó es aceptable y el 20\% afirmó, que está de acuerdo. Finalmente, el $40 \%$ de los encuestados afirmó estar de acuerdo con el perfil del egresado; de estos, el $6.7 \%$ estuvo en total acuerdo y el $33.3 \%$ estuvo de acuerdo con el plan de estudios. Por tanto, se rechaza la hipótesis nula y se acepta la existencia de una relación entre el plan de estudios y el perfil profesional del egresado de la Maestría en Agronegocios de la UNALM.

\section{Prueba de hipótesis específica N. 1.}

Hipótesis planteada: existe un nivel alto de congruencia del plan de estudios de la Maestría en Agronegocios de la UNALM, en función del perfil profesional del egresado.

Hipótesis nula: no existe un nivel alto de congruencia del plan de estudios de la Maestría en Agronegocios de la UNALM, en función del perfil profesional del egresado.

\section{En consecuencia:}

Se aprecia en la Tabla 4, que el nivel de congruencia de los objetivos institucionales está relacionada directamente con el plan de estudios 
de los egresados de la Maestría de Agronegocios de la UNALM, según la correlación de Spearman de 0.749 , representando una aceptable asociación de las variables y siendo altamente significativa. Además, según la prueba de independencia (Chi-cuadrado: $21.797 * * * p<.05)$ es altamente significativa. Por tanto, se acepta la existencia

Tabla 4. Congruencia con los objetivos institucionales

\begin{tabular}{|c|c|c|c|c|c|c|c|c|}
\hline \multirow{3}{*}{$\begin{array}{c}\text { Congruencia } \\
\text { de los } \\
\text { objetivos } \\
\text { institucionales }\end{array}$} & \multicolumn{6}{|c|}{ Variable: Plan de Estudios } & \multirow{2}{*}{\multicolumn{2}{|c|}{ Total }} \\
\hline & $\begin{array}{c}\text { En } \\
\text { desacuerdo }\end{array}$ & Aceptable & \multicolumn{2}{|c|}{ De acuerdo } & \multicolumn{2}{|c|}{$\begin{array}{l}\text { Totalmente } \\
\text { de acuerdo }\end{array}$} & & \\
\hline & $\mathrm{f} \quad \%$ & $\%$ & $f$ & $\%$ & $\mathrm{f}$ & $\%$ & $\mathrm{f}$ & $\%$ \\
\hline En desacuerdo & $1 \quad 6,7 \%$ &, $0 \%$ & 0 &, $0 \%$ & 0 &, $0 \%$ & 1 & $6,7 \%$ \\
\hline Aceptable &, $0 \%$ & $5 \quad 33,3 \%$ & 3 & $20,0 \%$ & 0 &, $0 \%$ & 8 & $53,3 \%$ \\
\hline De acuerdo &, $0 \%$ &, $0 \%$ & 5 & $33,3 \%$ & 1 & $6,7 \%$ & 6 & $40,0 \%$ \\
\hline Total & $6,7 \%$ & $533,3 \%$ & 8 & $53,3 \%$ & 1 & $6,7 \%$ & 15 & $100,0 \%$ \\
\hline $\begin{array}{l}\text { Chi-cuadrado } \\
\text { Correlación de }\end{array}$ & $\begin{array}{l}\text { Pearson }= \\
\text { earman }\end{array}$ & $\begin{array}{l}21.797 \\
749\end{array}$ & & $p<.05$ & & & & \\
\hline
\end{tabular}

de un alto nivel de congruencia del plan de estudios de la Maestría en Agronegocios de la UNALM, en función del perfil profesional del egresado.

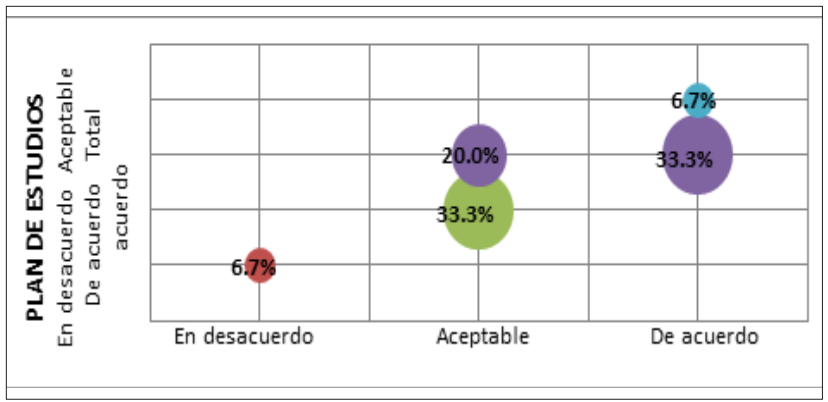

Figura 2. Congruencia objetivos institucionales y plan de estudios

Nota: en base a cuestionarios aplicados a la muestra del estudio, en el año 2013

\section{Prueba de hipótesis específica N. 2.}

Hipótesis planteada: existe un nivel alto de continuidad del plan de estudios de la Maestría en Agronegocios de la UNALM, en función del perfil profesional del egresado.

Hipótesis nula: no existe un nivel alto de continuidad del plan de estudios de la Maestría en Agronegocios de la UNALM, en función del perfil profesional del egresado.

\section{En consecuencia:}

Se aprecia en la Tabla 5, que el nivel de continuidad con el seguimiento de los graduandos está relacionada directamente con el plan de estudios de los egresados de la Maestría de Agronegocios de la UNALM, según la correlación de Spearman de 0.874, representando ésta una aceptable asociación de las variables y siendo altamente significativa. Además, según la prueba de independencia (Chi-cuadrado: $26.172 * * * p<.05)$ es altamente significativa. Por tanto, se acepta la existencia de un nivel alto de continuidad del plan de estudios de la Maestría en Agronegocios de la UNALM, en función del perfil profesional del egresado.

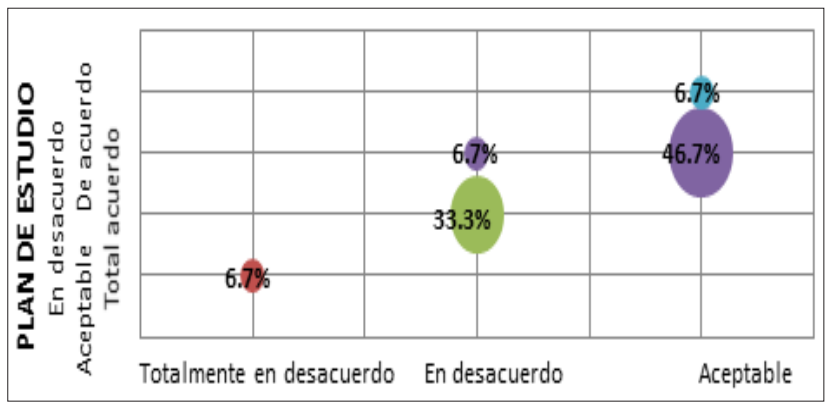

Figura 3. Continuidad: seguimientos a graduados y plan de estudios

Nota: en base a cuestionarios aplicados a la muestra del estudio, en el año 2013.
Se observa en la Figura 2, que el $6.7 \%$ de los encuestados afirmaron no estar de acuerdo con el nivel de congruencia de los objetivos institucionales y estar en desacuerdo con el plan de estudios de la Maestría de Agronegocios. Por otro lado, el 53.3\% de los encuestados afirmaron, que es aceptable el nivel de congruencia de los objetivos institucionales; de estos, el $33.3 \%$ planteó es aceptable y, el 20\% afirmó estar de acuerdo. Finalmente, el 40\% de los encuestados afirmó estar de acuerdo con el nivel de congruencia de los objetivos institucionales; de estos, el $6.7 \%$ estuvo en total acuerdo y el $33.3 \%$ estuvo de acuerdo. Por tanto, se rechaza la hipótesis nula y se acepta la existencia de un nivel alto de congruencia del plan de estudios de la Maestría.
Tabla 5. Comunidad: Seguimiento a graduados

\begin{tabular}{|c|c|c|c|c|c|c|c|c|c|c|}
\hline \multirow{3}{*}{$\begin{array}{l}\text { Continuidad: } \\
\text { Seguimiento a } \\
\text { graduados }\end{array}$} & \multicolumn{8}{|c|}{ Variable: Plan de Estudios } & \multirow{2}{*}{\multicolumn{2}{|c|}{ Total }} \\
\hline & \multicolumn{2}{|c|}{$\begin{array}{c}\text { En } \\
\text { desacuerdo }\end{array}$} & \multicolumn{2}{|c|}{ Aceptable } & \multicolumn{2}{|c|}{ De acuerdo } & \multicolumn{2}{|c|}{$\begin{array}{l}\text { Totalmente } \\
\text { de acuerdo }\end{array}$} & & \\
\hline & $f$ & $\%$ & $f$ & $\%$ & f & $\%$ & $\mathrm{f}$ & $\%$ & $\mathrm{f}$ & $\%$ \\
\hline Tota & 1 & $6,7 \%$ & 0 &, $0 \%$ & 0 &, $0 \%$ & 0 &, $0^{\circ}$ & 1 & $6,7 \%$ \\
\hline $\mathrm{nde}$ & 0 &, $0 \%$ & 5 & $33,3 \%$ & 1 & 6 , & 0 & , & 6 & $40,0 \%$ \\
\hline Aceptable & 0 &, $0 \%$ & 0 &, $0 \%$ & 7 & $46,7 \%$ & 1 & 6,7 & 8 & $53,3 \%$ \\
\hline Total & 1 & $6,7 \%$ & 5 & $33,3 \%$ & 8 & $53.3 \%$ & 1 & $6,7 \%$ & 15 & $100,0 \%$ \\
\hline
\end{tabular}

Chi-cuadrado de Pearson $=26.172 \quad$ g.l. $=6 \quad p<.05$

Correlación de Spearman $=.874$

Nota: en base a cuestionarios aplicados a la muestra del estudio, en el año 2013.

Se aprecia en la Figura 3, que el 6.7\% de los encuestados afirmó estar totalmente en desacuerdo con el nivel de continuidad del plan de estudios a través del seguimiento a los graduados. Asimismo, el 6.7\% estuvo en desacuerdo 
con el plan de estudios de la Maestría en Agronegocios. Por otro lado, el $40 \%$ de los encuestados afirmó, que es aceptable el nivel de continuidad desde el seguimiento a los graduados; de estos, el $33.3 \%$ planteó es aceptable el plan de estudios y, el 6.7\% afirmó estar de acuerdo. Finalmente, el $53.3 \%$ de los encuestados afirmó estar de acuerdo con el nivel de continuidad en el seguimiento de los graduados; de estos, el $6.7 \%$ estuvo en total acuerdo y, el $46.7 \%$ estuvo de acuerdo. Por tanto, se rechaza la hipótesis nula y se acepta la existencia de un nivel alto de continuidad del plan de estudios de la Maestría en Agronegocios, en función del perfil profesional del egresado.

\section{Prueba de hipótesis específica N. 3.}

Hipótesis planteada: existe un nivel alto de integración del plan de estudios de la Maestría en Agronegocios de la UNALM, en función del perfil profesional del egresado.

Hipótesis nula: no existe un nivel alto de integración del plan de estudios de la Maestría en Agronegocios de la UNALM, en función del perfil profesional del egresado.

\section{En consecuencia:}

Se aprecia en la Tabla 6, que el nivel de integración del perfil está relacionado directamente con el plan de estudios en los egresados de la Maestría de Agronegocios de la UNALM, según la correlación de Spearman de

Tabla 6. Dimención: Perfil publicado

\begin{tabular}{|c|c|c|c|c|c|c|c|c|c|c|}
\hline \multirow{3}{*}{$\begin{array}{c}\text { Integración: } \\
\text { Perfil } \\
\text { publicado }\end{array}$} & \multicolumn{8}{|c|}{ Variable: Plan de Estudios } & \multirow{2}{*}{\multicolumn{2}{|c|}{ Total }} \\
\hline & \multicolumn{2}{|c|}{$\begin{array}{c}\text { En } \\
\text { desacuerdo }\end{array}$} & \multicolumn{2}{|c|}{ Aceptable } & \multicolumn{2}{|c|}{ De acuerdo } & \multicolumn{2}{|c|}{$\begin{array}{l}\text { Totalmente } \\
\text { de acuerdo }\end{array}$} & & \\
\hline & $\mathrm{f}$ & $\%$ & $\mathrm{f}$ & $\%$ & $\mathrm{f}$ & $\%$ & $\mathrm{f}$ & $\%$ & $\mathrm{f}$ & $\%$ \\
\hline En desacuerdo & 1 & $6,7 \%$ & 0 &, $0 \%$ & 0 &, $0 \%$ & 0 &, $0 \%$ & 1 & $6,7 \%$ \\
\hline Aceptable & 0 &, $0 \%$ & 5 & $33,3 \%$ & 2 & $13,3 \%$ & 0 &, $0 \%$ & 7 & $46,7 \%$ \\
\hline De acuerdo & 0 &, $0 \%$ & 0 &, $0 \%$ & 6 & $40,0 \%$ & 1 & $6,7 \%$ & 7 & $46,7 \%$ \\
\hline Total & 1 & $6,7 \%$ & 5 & $33,3 \%$ & 8 & $53.3 \%$ & 1 & $6,7 \%$ & 15 & $100,0 \%$ \\
\hline $\begin{array}{l}\text { Chi-cuadrado } \\
\text { Correlación de }\end{array}$ & $P$ & $\begin{array}{l}o n=2 . \\
a n=.8\end{array}$ & $\begin{array}{l}571 \\
5\end{array}$ & g.l. $=$ & & $p<.05$ & & & & \\
\hline
\end{tabular}

0.805, representando ésta una aceptable asociación de las variables y siendo altamente significativa. Además, según la prueba de independencia (Chi-cuadrado: $23.571 * * * p$ $<.05)$ es altamente significativa. Por tanto, se acepta la relación entre el nivel de integración del plan de estudios de la Maestría en Agronegocios de la UNALM, en función del perfil profesional del egresado.

Se aprecia en la Figura 4, que el 6.7\% de los encuestados afirmó estar totalmente en desacuerdo con el nivel de integración del perfil publicado; de estos, el 6.7\% estuvo en desacuerdo con el plan de estudios. Además, el 46.7\% afirmó es aceptable el nivel de integración del perfil publicado; de estos, el 33.3\% afirmó es aceptable y el $13.3 \%$ estuvo de acuerdo con el plan de estudios. Finalmente, el $46.7 \%$ de los encuestados afirmó estar de acuerdo con el nivel de integración del perfil publicado; de estos, el 40\% afirmó estar de acuerdo y el 6.7\% planteó estar en desacuerdo. Por tanto, se rechaza la hipótesis nula y se acepta la existencia de un nivel alto de integración del plan de estudios de la Maestría en Agronegocios, en función al perfil profesional del egresado.

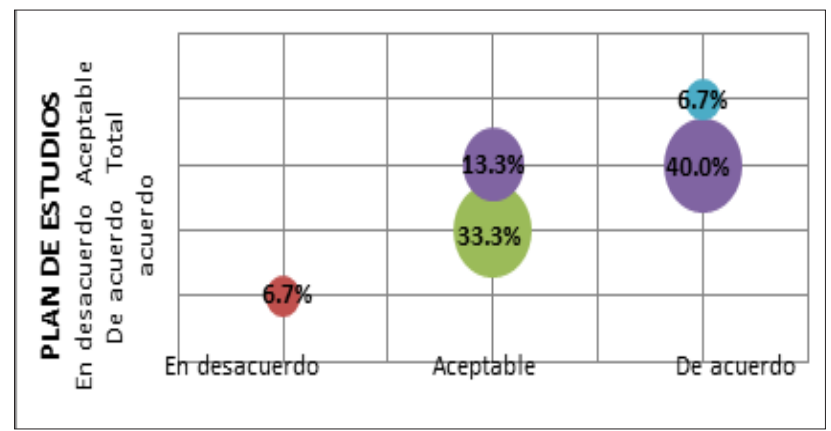

Figura 4. Integración: perfil publicado y plan de estudios. Nota: en base a cuestionarios aplicados a la muestra del estudio, en el año 2013

\section{Discusión.}

Las diversas teorías sobre el currículo evolucionan, son dinámicas, se encuentran en constante cambio como es la práctica curricular. En virtud a los antecedentes, se logró comprobar la existencia de una relación entre los planes de estudio y el perfil profesional de los egresados, que sirvió de base al objeto de estudio, entre ellas: Pereyra (2012), Llanos (2010), Barrientos y Vildoso (2007), Ramírez y Tovar (2005) y Rodríguez del Solar (1997) que demuestran la validez entre los planes de estudio y su relación con el perfil de los egresados de diversas instituciones de nivel superior.

La discusión de resultados desde las hipótesis planteadas contra los resultados obtenidos confirmaron la existencia de una relación entre el plan de estudios de la Maestría en Agronegocios de la UNALM y el perfil profesional de sus egresados, según la Tabla 3, y la correlación de Spearman y Chi-cuadrado, y en función a las teorías utilizadas, al haberse considerado el currículo como plan, siguiendo a Saylor y Alexander (1974) los cuales se enfocaron en los objetivos y los medios, pero que no se limitó sólo a eso, pues incluye, además, estrategias de instrucción y evaluación. El plan de estudios es el medio que selecciona y organiza los conocimientos con criterios de secuencialidad y continuidad, fundamentales para el logro del perfil académico profesional.

El perfil profesional es el conjunto de rasgos, capacidades y competencias que permiten al egresado ser reconocido por la sociedad peruana como tal profesional, encomendándole tareas para las que se le supone capacitado y competente. Diversos autores, entre ellos, Díaz (1981) opina, que el perfil profesional lo componen tanto conocimientos, habilidades y actitudes. Asimismo, autores como Tyler 
(1973), incluyen dentro del currículo algunos elementos comunes, tales como: conocimientos, valores, costumbres, creencias, hábitos. Las propuestas de los autores coinciden en la especificación de las habilidades, acciones o tareas que debe realizar el profesional para considerarse como tal. Autores como Díaz, Lule, Pacheco, antes citados, y otros respaldan y sugieren que los elementos a considerar dentro del perfil profesional, siendo integral en las dimensiones humanas involucradas en toda educación, pueden ser: dimensión cognoscitiva, actitudinal y destrezas, como se mencionó líneas arriba.

Siguiendo a Castro (1984), el modelo está diseñado para determinar la validez del diseño curricular de una institución, considerándose aquellos criterios que permitieron medir la validez interna y externa, bajo los criterios o niveles de congruencia, continuidad e integración. La congruencia es una relación de equilibrio y proyección existente entre los elementos iniciadores y orientadores con los indicadores correspondientes, como así mismo, los elementos de planificación y organización y los elementos de participación y administración. La continuidad es una relación de secuencia, interdependencia, alcance y articulación horizontal de los indicadores del Plan de Estudios, con los elementos generadores y, la integración es una interrelación, interdependencia y correspondencia de los objetivos, con los elementos generadores.

En ese sentido, la discusión de resultados desde la mirada de la hipótesis específica N. 1 planteada contra los resultados obtenidos confirman un alto nivel y muy significativo de congruencia del plan de estudios de la Maestría, en función del perfil del egresado, según la Tabla 4. La evaluación del nivel de congruencia de los objetivos institucionales está directamente relacionada con el plan de estudios en los egresados y del perfil profesional en relación con los fundamentos de la carrera. Esta valoración se realizó al buscar el grado en que el perfil es una consecuencia lógica de lo fundamentado; es decir, que el perfil profesional responde a los resultados de la presente investigación y de las anteriores realizadas, al amparo de su propia fundamentación.

La discusión de resultados desde la mirada de la hipótesis específica N. 2 planteada contra los resultados obtenidos confirman un alto nivel y altamente significativo de continuidad del plan de estudios de la Maestría, en función del perfil del egresado, según la Tabla 5. Díaz, F., Lule, M., Pacheco, D., Rojas, S. y Saad, E. (2011) establecen que el proceso de evaluación curricular debe ser continuo, incidiendo en todas las etapas del proceso curricular, y flexible, atendiendo tanto a consecuencias esperadas, como no anticipadas. Sin embargo, aquí también se ve altamente relacionado el seguimiento al perfil del egresado, sin el cual no se podrían lograr los objetivos institucionales, como parte del sistema o los elementos que forman parte del currículo.

La discusión de resultados desde la mirada de la hipótesis específica N. 3 planteada contra los resultados obtenidos describen un nivel altamente significativo de integración del plan de estudios de la Maestría, en función del perfil del egresado, según la Tabla 6.

\section{Conclusiones}

Se logró demostrar, según los resultados obtenidos, que sí existe relación entre el plan de estudios de la Maestría en Agronegocios de la UNALM, en función al perfil profesional de sus egresados que impacta favorablemente en el logro de sus conocimientos, actitudes y valores y habilidades en la comunidad. Se logró determinar que sí existe un alto nivel de congruencia, de continuidad y de integración del plan de estudios de la Maestría, en función al perfil profesional sus egresados. Respecto a los objetivos institucionales, el $60 \%$ de egresados planteó son aceptables y el $40 \%$ estuvo de acuerdo. Respecto al seguimiento de graduados, el 53.3\% lo considero aceptable. Respecto al perfil publicado, el 93.3\% afirmó es aceptable y estar de acuerdo. Respecto al perfil del profesional egresado, el 86.6\% afirmó es aceptable y estar de acuerdo. Respecto al aspecto formativo, actitudinal y cognitivo del plan de estudios, los egresados los encontraron aceptables, en general.

\section{Recomendaciones}

En función a los resultados se deben corregir las distorsiones o debilidades que puedan encontrarse $\mathrm{y}$ fortalecer las buenas prácticas que posee el programa. Se debe prestar particular atención al seguimiento a sus graduados y los aspectos formativo, actitudinal, personal y cognitivo del plan de estudios. Además, a) realizar evaluaciones periódicas al plan de estudios y al perfil del egresado de la Maestría en Agronegocios de la UNALM, para actualizar los criterios educativos manejados por la institución; b) generar y administrar una matriz mucho más completa para garantizar la formación integral del futuro egresado, en actitudes y destrezas que le permitirán desenvolverse en forma eficaz y eficiente en el desempeño de su profesión; c) conformar un equipo interdisciplinario que evalué y fortalezca el plan de estudios y el perfil del egresado de la Maestría; d) generar líneas de investigación sobre la relación existente entre el plan de estudios de la maestría y el perfil profesional de sus egresados acordes a las necesidades del mercado; e) conformar una Comisión Revisora del plan de estudio de la maestría y del perfil de sus egresados, a fin de elevar la calidad y el sostenimiento de la educación impartida en la Maestría en Agronegocios de la UNALM.

\section{Literatura citada}

Albornoz, O. 1991. La Universidad que Queremos. Caracas: Ed. de la Biblioteca UCV.

Álvarez de Zayas, C. 2001. El Diseño Curricular. La Habana: Ed. Pueblo y Educación.

Arista, G. 1984. Metodología de la Investigación. Lima: Ed. EDUSMP. 
Barrientos, E. y Vildoso J. 2007. Influencia del perfil académico y el plan de estudios en el nivel de conocimiento de investigación en los maestristas de la unidad de post grado en la Facultad de Educación. Tesis Mg. Facultad de Educación de la Universidad Nacional Mayor de San Marcos. Lima: UNMSM.

Castro, M. 1984. Modelo de desarrollo, control y ajuste permanente del curriculum. Universidad de Toulouse, París: Le Mirail.

Díaz, Á. 1981. Alcances y limitaciones de la metodología para la realización de planes de estudio. Centro de Estudios Educativos. México: Revista de Educación Superior, n 40.

Díaz, Á. 1993. Diseño curricular II. México: ILCE.

Díaz, F., Lule, M., Pacheco, D., Rojas, S. y Saad, E. 2011. Metodología de Diseño Curricular para educación superior. México: Ed. Trillas.

Hernández, R. 1996. Metodología de la investigación. Bogotá: Ed. McGraw Hill.

Hernández, R., Fernández, A. y Baptista, M. 2003. Tipos de investigación. México: McGraw Hill.

Hernández, R., Fernández, A. y Baptista, M. 2006. Metodología de la Investigación. 4ta edición. México: Ed. McGraw-Hill Interamericana.

Kissack, J. 1998. Conferencia, sobre relación entre la Investigación y el Currículo y la Ética en la Investigación. Material de lectura; Unidad de Post Grado. Lima: Facultad de Educación, Universidad Nacional Mayor de San Marcos.

Llanos, J. 2010. Relación del nivel de relación entre el perfil profesional y el Plan de estudios de la Facultad de Ciencias de la Educación de la Universidad Nacional Hermilio Valdizán de Huánuco. Tesis doctoral de la Facultad de Ciencias de la Educación de la Universidad Nacional Hermilio Valdizán.

Pagano, R. 1999. Estadística para las ciencias del comportamiento. México: Editorial Thomson.

Pereira, J. 2012. La formación profesional y el mercado laboral de los técnicos agrícolas del Instituto Federal de Educación, Ciencia y Tecnología del Estado de Maranhão, Campus São Luís - Maracanã, Maranhão, Brasil. Tesis doctoral del Departamento de Didáctica del Programa de Doctorado la Acción Educativa: Perspectivas Históricas y Funcionales de la Universidad de Alcalá.

Ramírez, R. y Tovar, M. 2005. Egresados de las Especializaciones en Enfermería. Evaluación de una década. Escuela de Enfermería e Instituto de Educación y Pedagogía de la Universidad del Valle. Tesis doctoral de la Escuela de Enfermería e Instituto de Educación y Pedagogía de la Universidad del Valle de Cali.

Rodríguez del Solar, N. 1997. Diagnóstico Curricular de la Facultad de Educación de la Universidad Nacional Mayor de San Marcos y propuesta de un perfil profesional con perspectiva andragógica. Tesis doctoral de la Facultad de Educación de la Universidad Nacional Mayor de San Marcos.

Salinas, D. 1994. La planificación de la enseñanza: ¿Técnica, sentido común o saber profesional. En: Teoría y Desarrollo del Currículo de Angulo y Blanco. Málaga: Ediciones Aljibe.

Saylor, J. \& Alexander, W. 1974. Planeamiento del Currículo en las Escuelas Modernas. Buenos Aires: Ed. Troquel S. A.

Tyler, R. 1973. Principios básicos del Curriculum. Buenos Aires: Ed. Troquel.

Zabalza, M. 2012. Articulación y rediseño curricular: el eterno desafío institucional. Revista de Docencia Universitaria, 10 (3): 17 - 48. Santiago de Compostela, España. 